\title{
QUANTO INFLUISCONO I SISTEMI ELETTORALI
}

SULLE CAMPAGNE? UN'ANALISI COMPARATA IN NOVE STATI EUROPEI

di FranCESCA Montemagno e Rossana SAMPUGNARO 


\begin{abstract}
The demand for specific expertise to manage strategically election campaigns is growing. However, their use depends on the history of political party, on their values and on their economic resources and to the context in which the election campaign takes place. In this sphere, the electoral system produces constraints and incentives for the development of the electoral campaigns.

This study aims to examine the influence of electoral system on electoral campaign style and on its management. In particular, the focus is on professionalization and personalization of electoral campaigns within a comparative approach among the European states. The data are from a comparative study on candidates (CCS).

The study focuses on the candidates who participated in the most recent general elections included in the database. In particular, we selected nine countries with different electoral systems: for Candidate-based electoral system, we choice Romania, United Kingdom, Ireland and Malta; for Party-based electoral system, we examined Norway, Portugal and Italy; we also included in our analysis Hungary and Germany, which have a Mixed system with single-member constituency and closed List, which can be placed between the two previous systems.

The results of the study show that the electoral systems influence the election campaigns of candidates, producing different models of mobilization.
\end{abstract}

Keyword: electoral system, general elections, electoral campaign 


\section{Le campagne elettorali e il ruolo del sistema elettorale}

Le campagne elettorali sono nei loro caratteri essenziali un'organizzazione (Farrell e Webb, 2002) e una strategia di comunicazione necessaria per la mobilitazione del proprio elettorato o per la smobilitazione di quello degli avversari (Cox, 1999; Karp et al., 2007; Strömbäck e Kiousis, 2014). Nel 2001 Johnson dichiarava che le campagne erano «non place for amateurs» prospettando un radicale cambiamento delle strutture dedite alla campagna, nelle quali ben poco spazio rimaneva per i semplici volontari o per i supporter del candidato. Nel tempo si è realizzata una professionalizzazione dell'organizzazione con un rafforzamento della domanda di servizi specialistici da parte di partiti e candidati: esperti di media, sondaggisti, campaign directors, e in ultimo, social media managers e analisti dei big data.

Nel passaggio al web campaigning si rafforza ulteriormente la tendenza a costruire le strategie di comunicazione sui candidati e sui leader al posto dei partiti. La personalizzazione della politica risponde alla necessità di costruire un discorso politico più efficace rispetto a quello possibile su organizzazioni come i partiti: i dibattiti televisivi, le campagne politiche e i media event si concentrano sulle caratteristiche e sulle performance dei singoli uomini politici (Poguntke e Webb, 2005). In questi ultimi anni, la digitalizzazione delle campagne ha favorito ulteriormente la personalizzazione: attraverso account personali sui social networks, i candidati comunicano e costruiscono relazioni con singoli elettori che diventano coproduttori di servizi (programmi, messaggi, slogan). In questo nuovo contesto (Bennett e Segelberg, 2012), il politico sollecita direttamente i suoi supporters senza nessuna mediazione partitica.

Questi due fondamentali elementi - professionalizzazione e personalizzazione delle campagne ${ }^{1}$ - variano in relazione alla storia delle formazioni politiche, ai loro orientamenti valoriali ed in funzione delle loro risorse (Sampugnaro, 2010). Ne consegue che i servizi specialistici richiesti

\footnotetext{
1 Anche se il termine professionalizzazione richiederebbe un'adeguata storicizzazione (Panebianco, 1982), in questo contesto viene utilizzata una sua accezione più recente che attiene all'inserimento nelle organizzazioni di partito e nei comitati elettorali di professionisti della mobilitazione, della comunicazione e dei sondaggi (più estesamente in Sampugnaro, 2006; vedi anche Mancini, 2015; Negrine et al., 2007). La personalizzazione è qui ricondotta ad una dimensione comunicativa (personalizzazione della politica) che non contempla necessariamente un processo di concentrazione del potere (personalizzazione del potere o presidenzializzazione) (Bordignon, 2014; Calise, 2010).
} 
dai partiti e dai candidati possono assumere - specie nella fase di accreditamento di alcune competenze - caratteri molto diversi e che la variabilità è elevata se compariamo l'evoluzione nei singoli Stati (Strömbäck e Kiousis, 2014), o anche limitandola alla sola Europa (Tenscher et al., 2015). L'accentuazione di questi tratti dipende dalla tipologia di competizione elettorale e può essere influenzata anche da elementi sistemici o contestuali (una rassegna in Esser e Strömbäck, 2012), come una particolare (e anche contingente) struttura della competizione o la presenza di un determinato sistema elettorale.

Quest'ultimo si conferma come un fondamentale elemento esplicativo delle strategie degli attori politici direttamente coinvolti in attività di mobilitazione diretta e di quelli (sindacati, associazioni di categoria, gruppi di interesse) che si attivano per la mobilitazione secondaria (Cox 2015, p. 65). Il quadro normativo condiziona le scelte di partiti e candidati (Carey e Shugart, 1995) perché costituisce un sistema di incentivi e di limiti per le loro attività in una prospettiva di breve e di lungo periodo, anche se bisogna sottolineare la necessità di tenere in considerazione altri fattori contestuali come le caratteristiche della competizione, il sistema dei partiti o le peculiari tradizioni politiche (Tenscher e Mykkänen, 2014).

Senza pretesa di esaustività in questa sede, di questi elementi è utile fornire talune esemplificazioni. Se guardiamo alla competizione dal punto di vista dei partiti (Lago et al., 2017; Denemark, 2003; Karp, 2012), la magnitudo dei collegi influisce sulle scelte di intensificazione delle attività elettorali, inducendo a un diverso investimento in termini umani e monetari sulle singole circoscrizioni elettorali. A parità di sistema elettorale, un'alta polarizzazione politica disincentiva il contatto diretto con i votanti che diventa invece fondamentale quando lo spazio politico è più ristretto e gli elettori possono essere "convertiti" con più facilità (Karp 2012, p. 87). Se guardiamo ai singoli politici, il sistema elettorale non determina solo le scelte strategiche del candidato nel corso della campagna ma - limitatamente agli eletti - condiziona anche lo "stile" di rappresentanza nelle assemblee legislative. È noto, ad esempio, che i sistemi proporzionali producano un consistente livello di competizione intrapartitica nelle sedi parlamentari: i deputati sono indotti a "personalizzare" parte delle attività parlamentari perché cercano di capitalizzare una visibilità che li aiuterà ad essere rieletti (Müller e Strøm, 1999; Russo, 2013; Pinto e Verzichelli, 2016; Camatarri e Segatti, 2016). Quanto più "individuale" è la concezione della rappresentanza, tanto maggiore sarà l'interpretazione personalistica della stessa campagna elettorale (Autori dell'articolo 2017). 
Il sistema elettorale è un tassello tanto fondamentale nella competizione elettorale da essere, prima ancora di un sistema di constraints per la campagna elettorale (variabile indipendente), un ambito di aperta competizione tra i partiti per definizione delle regole che possono migliorare il vantaggio della singola formazione alla ricerca del consenso (Colomer, 2017). L'ambito prescelto per il nostro studio è quello che vede il sistema elettorale come un vincolo esterno, interrogandosi su come possa condizionare lo stile della campagna elettorale del singolo candidato, le sue strategie comunicative o più in generale la sua conduzione. Nello specifico la ricerca indaga sulla relazione tra le regole elettorali che si applicano alle elezioni politiche e le caratteristiche delle campagne elettorali dei singoli candidati. Pur ben consapevoli della complessità delle variabili in gioco e di un numero limitato di casi nazionali, lo studio ha uno scopo esplorativo tendente ad individuare dei modelli di campagna attraverso l'associazione di modalità di variabili.

I sistemi elettorali sono stati oggetto di attenzione da parte di molti studiosi che, oltre ad averli classificati, ne hanno valutato gli effetti e le influenze (Clark et al., 2011). Le regole che governano le competizioni elettorali, oltre a consentire una traduzione dei voti in seggi sulla base dall'ampiezza del collegio, dalla formula elettorale e dalla soglia di rappresentanza (Lijphart, 1994), condizionano e regolano, come ricorda Cox, la mobilitazione e la competizione intrapartitica ed interpartitica (1999, p. 387).

Volendo restringere il campo delle influenze di questi sistemi alle sole campagne elettorali, possiamo fare un'iniziale distinzione tra candidate-based electoral systems e party-based electoral systems (Plasser e Plasser, 2002).

I primi, tra i quali possiamo includere i sistemi elettorali maggioritari basati su liste plurinominali aperte, sul voto singolo trasferibile o sul principio del first past the post, favoriscono campagne elettorali decentralizzate, individualizzate, personalizzate (specie in presenza di collegi plurinominali con liste aperte) e sottolineano le qualità e le caratteristiche del singolo candidato. La possibilità per l'elettore di esprimere una preferenza porta i candidati a scegliere una gestione della campagna elettorale che assume sempre più la fisionomia di una horse-race: la competizione si gioca non solo tra candidati di partiti diversi, ma anche tra candidati dello stesso partito. Queste campagne elettorali, i cui tempi sono notevolmente dilatati, sono inoltre sempre più caratterizzate dalla presenza di staff professionalizzati. Con la sua campagna elettorale il candidato mirerà alla costruzione di un supporto personale. La performance, 
le capacità ed il profilo del candidato, acquisiscono sempre maggiore importanza.

I party-based electoral systems, diversamente, sono caratterizzati generalmente da liste bloccate. Queste ultime sono costruite dal gruppo dirigente di partito con metodologie che prevedono diverse forme di coinvolgimento del selettorato e vari criteri di selezione delle candidature e dell'ordine delle posizioni in lista (es: primarie o selezione interna). In generale questi sistemi hanno la tendenza a produrre campagne elettorali centralizzate, di ampiezza nazionale e centrate sul partito. In questo specifico caso, la vittoria del candidato è determinata esclusivamente dalla forza della lista cui appartiene e di conseguenza viene meno la necessità di costruire un rapporto duraturo con l'elettore o concentrarsi sui temi locali e sui i problemi del territorio. In queste competizioni, per il candidato diventa importante sostenere il programma di partito e "sventolare la sua bandiera".

Questa divisione però può apparire limitata se consideriamo l'esistenza di modelli intermedi (mixed-members systems) che si sono largamente diffusi, con motivazioni diverse (Carter e Farrell, 2010) nelle "new democracies" e in quelle consolidate. In queste ultime, la loro adozione può essere ricondotta a motivazioni che cercano di controbilanciare le tendenze naturali dei due sistemi elettorali: quello maggioritario, che produce prima candidati e poi eletti tanto forti da sfuggire alla volontà dei partiti, o quello proporzionale che, nella sua versione pura e con le liste bloccate, esalta il ruolo accentratore del partito e riduce l'importanza del singolo candidato. Possiamo considerare i primi due come punti estremi di un ideale continuum, gli altri invece, come sistemi intermedi, che combinano seggi espressione di competizioni in collegi uninominali, con seggi frutto di attribuzione proporzionale di cariche elettive, in base a liste di partito concorrenti (Shugart e Wattenberg, 2001; Shugart 2001, p. 173).

La ricaduta dei sistemi elettorali sulle campagne dovrebbe condurre a modelli molto differenti nella pratica, almeno per ciò che riguarda i singoli candidati nei collegi. Se consideriamo la campagna dei leader dei singoli partiti, lo scivolamento verso una personalizzazione dei messaggi è evidente anche nei sistemi basati sul partito, oltre che per l'opportunità di interazione immediata che le nuove tecnologie consentono (Kreiss, 2015).

Diversa cosa è considerare il contributo alla campagna elettorale del candidato nel collegio uninominale o plurinominale. A questo livello è possibile distinguere le attività personali e quelle riconducibili al partito, considerando nel primo caso la presenza di una struttura di campagna autonoma in termini funzionali e di una serie di attività riconducibili al 
singolo. Partendo da questo quadro teorico, lo scopo di questo lavoro è quello di individuare le caratteristiche peculiari delle campagne dei candidati alle elezioni politiche, tenendo conto dello stato europeo d'appartenenza e quindi del peculiare sistema elettorale adottato per l'elezione dei membri dei Parlamenti nazionali.

L'ipotesi è che i sistemi elettorali siano in grado di condizionare le campagne elettorali, la loro durata, i contenuti e il loro livello di personalizzazione e professionalizzazione.

\section{Le scelte metodologiche}

Al fine di verificare il ruolo del sistema elettorale sui caratteri della professionalizzazione e personalizzazione delle campagne elettorali con un approccio comparativo tra gli stati europei, sono stati utilizzati dati raccolti nell'ambito del progetto di ricerca Comparative Candidates Survey (CCS). Il CCS è un'indagine multinazionale che ha l'obiettivo di raccogliere dati sui candidati che partecipano in diversi Paesi alle elezioni dei Parlamenti nazionali. Questa indagine utilizza un questionario comune tale da garantire un'adeguata analisi comparata. La raccolta dati include inoltre informazioni contestuali riguardanti la circoscrizione/collegio elettorale del candidato e il sistema politico del Paese.

Questo progetto di ricerca, iniziato nel 2005, ha finora riguardato trentasei elezioni parlamentari. Questi dati sono stati archiviati e messi a disposizione dello Swiss Centre of Expertise in the Social Sciences FORS.

Attualmente il set di dati comprende un totale di 32 elezioni di 24 diversi Paesi: Australia, Austria, Belgio, Canada, Repubblica Ceca, Danimarca, Estonia, Finlandia, Germania, Grecia, Ungheria, Islanda, Irlanda, Italia, Lussemburgo, Malta, Olanda, Nuova Zelanda, Norvegia, Portogallo, Romania, Svezia, Svizzera e Regno Unito. Il questionario del CCS vuole analizzare le relazioni tra il candidato, il partito e gli elettori. La batteria di domande che lo compongono include la campagna elettorale del candidato, i meccanismi di selezione delle candidature, le questioni ideologiche, la sezione dedicata alla democrazia e alla rappresentanza, la biografia, il background politico e le attività del candidato. Il nostro interesse si limita ai Paesi che appartengono al continente Europeo. La nostra indagine si concentra sui candidati che hanno partecipato alle più recenti elezioni politiche incluse nel database e sulla sezione del questionario dedicata alle loro attività di campagna elettorale. In particolare 
abbiamo selezionato 9 Paesi $^{2}$ che presentano sistemi elettorali differenti: per i candidate-based electoral systems, abbiamo scelto Romania and Regno Unito (Single Member District Plurality with Single-member constituency), Irlanda e Malta (Single Transferable Vote); per i Party-based electoral systems, prenderemo in esame Norvegia, Portogallo ed Italia (List Proportional Representation with Closed List); inoltre abbiamo incluso nella nostra analisi i casi dell'Ungheria e della Germania, che presentano un sistema Mixed Dependent (o Mixed Member Proportional) con Singlemember constituency e Closed List, che potremmo definire a cavallo tra i due (Bormann e Golder, 2013) ${ }^{3}$.

La scelta è ricaduta su questi Paesi per ragioni legate al loro sistema di elezione dei Parlamenti. Essendo interessati a comprendere se vi sia una relazione tra le modalità di voto e la gestione della competizione elettorale, abbiamo individuato quei Paesi che presentavano da un lato dei sistemi elettorali candidate-based e dall'altro Paesi con Party-based electoral systems: dei 24 Stati inclusi nel database CCS, solo il Portogallo, l'Italia e la Norvegia utilizzarono negli anni della rilevazione un sistema elettorale proporzionale con liste bloccate. In questo caso la scelta è stata obbligata; per la stessa ragione sono state scelte Germania ed Ungheria per analizzare i contesti con la presenza di sistemi elettorali misti; diversamente per il gruppo di Paesi con candidate-based electoral system si è operata una scelta più ragionata. Da un lato eravamo interessati a valutare il comportamento di candidati che operano in contesti in cui vi sono alte influenze di partito ma forti incentivi alla personalizzazione e per questo abbiamo orientato la nostra scelta su quei Paesi che presentano Single-member constituency, dove, da una parte, il partito controlla e decide le candidature nel collegio e, dall'altra, si verifica una forte competizione interpartitica tra singoli candidati ${ }^{4}$. Dall'altro volevano rilevare la professionalizzazione e la personalizzazione della campagna elettorale laddove il sistema adottato

\footnotetext{
${ }^{2}$ Nel dettaglio presentiamo per Paese il numero dei casi e l'anno di elezione di riferimento: Regno Unito 1472 candidati (2010), Romania 407 candidati (2012), Irlanda 1186 candidati (2007), Malta 42 candidati (2013), Portogallo 257 candidati (2011), Norvegia 1011 candidati (2009), Italia 672 candidati (2013), Germania 789 candidati (2009) ed Ungheria 431 candidati (2010).

${ }^{3}$ Le informazioni su tipo di sistema elettorale e sul tipo di voto dei Paesi della nostra selezione sono state estratte da un database utilizzato in: Bormann, Nils-Christian e Matt Golder (2013). Il set di dati contiene informazioni su 1197 elezioni politiche e presidenziali che hanno avuto luogo in 433 Paesi democratici dal 1946 al 2011. Le informazioni relative a Italia (2013), Romania (2012) e Malta (2013) sono di nostra elaborazione.

${ }^{4}$ Regno Unito e Romania sono, anche in questo caso, gli unici Paesi del dataset che utilizzarono un sistema elettorale con Single - member constituency.
} 
genera una competizione intrapartitica oltre che interpartitica tra i candidati. Il single transferable vote è l'unico sistema elettorale proporzionale che non utilizza liste di partito. Questo sistema applica il voto di preferenza in collegi plurinominali. Gli elettori di solito, indicando un numero accanto al nome dei candidati, specificano se questi sono la loro prima, seconda, terza scelta e così via. In questo caso, dunque, l'elettore, non solo sceglie i propri candidati, ma addirittura esprime un ordine di preferenze. Questo sistema risulta essere in questo senso quello maggiormente centrato sul candidato, andando addirittura oltre gli effetti di personalizzazione della campagna che può generare un sistema elettorale che presenta liste aperte o flessibili ${ }^{5}$. Per questa ragione si è scelto di selezionare Malta ed Irlanda, gli unici Paesi del dataset CCS che utilizzano il single transferable vote per le elezioni generali.

Presentiamo qui di seguito la Figura 1 riepilogativa dei tipi di voto di ciascun Paese selezionato. Questi sono stati disposti secondo un preciso ordine che, a nostro parere, va dai sistemi maggiormente centrati sul candidato a quelli fortemente centrati sul partito.

\footnotetext{
${ }^{5}$ Per le elezioni generali oggetto della rilevazione del dataset CCS Austria (2008), Belgio (2010), Repubblica Ceca (2006), Danimarca (2011), Islanda (2009), Olanda (2006) e Svezia (2010) utilizzarono List Proportional Representation - Flexible List; Estonia (2011), Finlandia (2011), Grecia (2012) e Svizzera (2011) utilizzarono List Proportional Representation - Open list.
} 


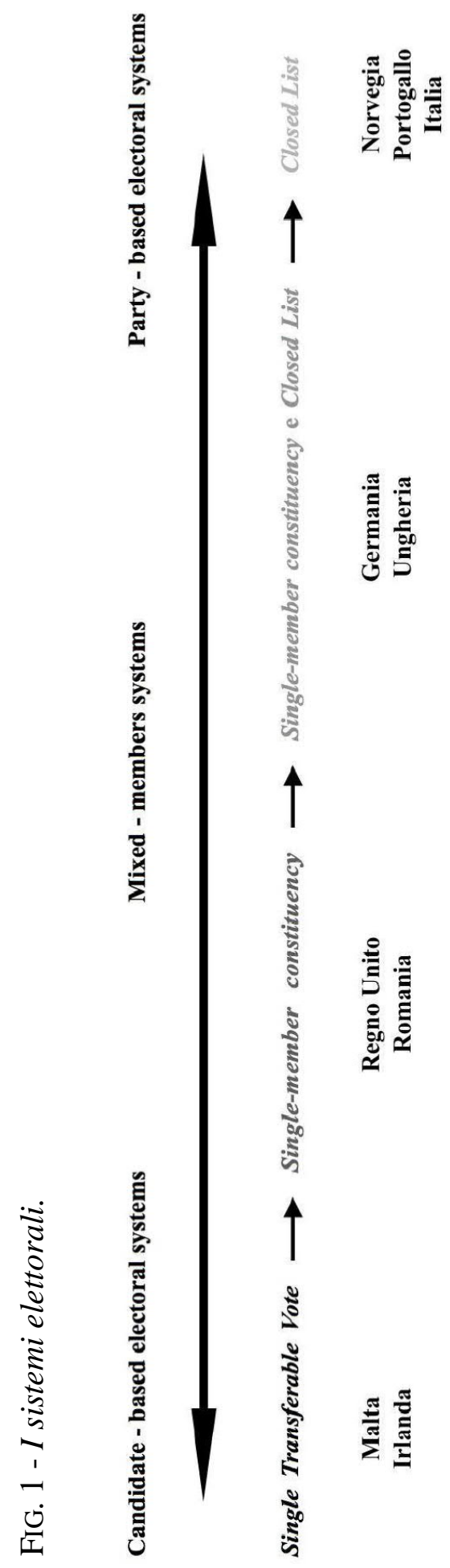


Per verificare quanto il tipo di sistema elettorale possa condizionare la gestione della campagna elettorale dei candidati, e in particolare il livello di professionalizzazione e personalizzazione della stessa, sono stati analizzati precedenti strumenti analitici, utilizzati per la ricerca comparativa. Riguardo alla formazione degli indicatori di professionalizzazione (Tenscher et al., 2012; Gibson e Römmele, 2001), si è operata una prima suddivisione tra elementi attinenti all'organizzazione e quelli riguardanti la strategia di campagna. Su questa base sono stati selezionati all'interno del questionario CSS e con i limiti derivanti dall'utilizzo dei dati secondari, quegli items ritenuti idonei a rappresentare questi due aspetti della gestione della campagna elettorale dei candidati.

Per rilevare la professionalizzazione della campagna elettorale abbiamo costruito un indice di tipo sommatorio calcolato utilizzando alcune variabili a loro volta semplificate attraverso l'aggregazione di alcune loro modalità:

- Organizzazione: variabili relative allo staff del candidato

1. Own personal campaign team. Questa variabile differenzia i candidati che hanno uno staff personale da coloro che non lo hanno. La presenza di uno staff personale, specie se caratterizzato dalla presenza di esperti e consulenti, è indicatore di professionalizzazione delle campagne elettorali. Abbiamo attribuito il valore 0 a chi dichiarava di non possederlo, 1 a chi indicava di esserne dotato.

2. Size of candidate Staff. Questa variabile indica il numero dei componenti dello staff personale del candidato. Il numero include sia il personale pagato dallo stesso candidato, sia quello pagato dal partito. Abbiamo semplificato il dato creando tre modalità ed includendo nella prima tutti i candidati che hanno dichiarato di non avere uno staff, nella seconda coloro i quali hanno risposto di possedere uno staff composto da almeno 7 componenti, nella terza coloro che hanno dichiarato di disporre di uno staff ampio, composto da più di 7 membri. Le campagne professionalizzate tendono ad avere degli staff particolarmente ampi e caratterizzati dalla presenza di personale specializzato, è per questo che ad ognuna di queste modalità abbiamo dato un peso differente, rispettivamente 0,1 e 2 .

3. Professional consultant. Questa variabile rileva la presenza di consulenti politici nella war room del candidato. Abbiamo attribuito il valore 2 a chi li possedeva, 0 a chi indicava di non disporre di personale specializzato. Abbiamo voluto dare un maggiore peso a questa variabile, essendo questa più delle altre indicatore di professionalizzazione della campagna elettorale. 
- $\quad$ Strategie: i tempi di campagna elettorale

1. How long before the election did you begin to organize your campaign?. L'intervistato poteva scegliere tra 5 diverse modalità: more than a year, 9-12 months, 6-9 months, 3-6 months e less than 3 months. Questa variabile è importante, in quanto i tempi delle campagne elettorali postmoderne si sono allungati, tanto da diventare addirittura permanenti. È per questo che abbiamo dato un peso maggiore a quelle campagne iniziate molto tempo prima rispetto al giorno delle elezioni. La pianificazione strategica di una campagna elettorale richiede tempi lunghi, è per questo che attribuiamo valore 3 a quei candidati che hanno risposto di aver iniziato la campagna elettorale fino a 9 mesi prima del voto; valore 2 a chi ha iniziato ad organizzarla da 9 a 3 mesi prima; valore 1 a chi ha iniziato solo 3 mesi prima del voto.

2. How long before the election did you start campaigning fulltime?. Le modalità di risposta proponevano la suddivisione in ore che ritroviamo nella variabile precedente. Anche in questo caso abbiamo pesato i tempi in maniera differente, seguendo la medesima codifica.

3. Hours devoted to campaigning at week during the last month before of the election. Per semplificare questo dato abbiamo aggregato le modalità per numero di ore dando un valore maggiore a chi dedicava più tempo alla propria campagna elettorale. Ai candidati che hanno detto di dedicare fino a 12 ore alla settimana alla propria campagna elettorale abbiamo dato valore 1; da 13 a 40 ore settimanali, abbiamo dato valore 2; superiore alle 40 ore abbiamo dato valore 3 .

Successivamente abbiamo calcolato il livello di professionalizzazione della campagna di ogni candidato sommando i loro valori in ciascuna variabile che costituiva l'indice. Ciascuna campagna poteva assumere un valore dell' indice che andava da un minimo di 3 (basso livello di professionalizzazione) ad un massimo di 14 (alto livello di professionalizzazione).

Per rilevare la personalizzazione della campagna elettorale del candidato, abbiamo scelto due indicatori: il primo era volto a verificare quanto i candidati avevano caratterizzato il loro profilo personale o quanto diversamente avevano centrato la propria campagna elettorale sul proprio partito di appartenenza. In questo caso l'intento è quello di cogliere l'individualizzazione e la leaderizzazione della competizione; il secondo invece voleva soffermarsi sulla personalizzazione dei contenuti della campagna e il recupero dei temi locali rispetto ai temi affrontati dal partito a livello nazionale ${ }^{6}$.

\footnotetext{
${ }^{6} \mathrm{Nel}$ dettaglio: 1. What was your primary aim during the campaign? Where would you place yourself on a scale from 0 to 10, where 0 means "to attract as much attention as
} 
Nello studio queste variabili e i relativi indici verranno valutati considerando lo stato di appartenenza e lo specifico sistema elettorale.

\section{Campagne elettorali a confronto: professionalizzazione e personaliz- zazione}

Lo studio sulle campagne elettorali è stato realizzato sui candidati ai Parlamenti nazionali dei 9 Paesi della nostra selezione. Prima di passare all'analisi dei risultati si ritiene opportuno presentare brevemente il profilo socio anagrafico e politico dei nostri candidati che evidenzia delle peculiarità nazionali (cfr. Tab.1). Il campione si presenta così caratterizzato: vi è una più alta percentuale di uomini rispetto alle donne. Il Paese in cui questa differenza appare più netta è l'Ungheria, dove solo il $10 \%$ della selezione è donna, quello che presenta una distribuzione di genere più equilibrata è la Norvegia, dove le donne raggiungono il $45 \%$ della selezione; la classe dei candidati alle general elections risulta piuttosto giovane. Il 36\% della selezione non supera i 45 anni di età. Anche in questo caso l'Ungheria si distingue dalle altre per la presenza di una classe politica più anziana, ben 1'84\% degli intervistati supera i 56 anni d'età. Il Paese con una percentuale maggiore di candidati giovani è la Romania, seguita dal Portogallo; il livello d'istruzione presentato in Tabella 1 si riferisce alla percentuale di candidati che hanno raggiunto un livello di istruzione universitaria. L'Irlanda e la Germania non presentano questo dato. La Romania, il Portogallo e Malta sono i Paesi che nell'ordine presentano le percentuali più alte di candidati laureati. Nessun candidato ungherese ha conseguito un titolo di studio universitario; 1'80\% dei candidati ai Parlamenti ha un'occupazione. La percentuale di occupati più bassa la ritroviamo in Germania. Questa informazione non è stata rilevata in Ungheria, Malta e Romania; solo il 12, $50 \%$ dei candidati della nostra selezione è stato precedentemente eletto. La presenza di incumbent è maggiore in Portogallo. In Ungheria, Italia e Regno Unito maggiore è la presenza di nuove leve; osservando con maggiore dettaglio la carriera politica dei candidati intervistati, notiamo che solo il $13 \%$ di loro, prima di candidarsi per il Parlamento, ha lavorato nello staff di

possible for me as a candidate" and 10 means "to attract as much as possible attention for my party?". Per semplificare abbiamo aggregato i valori in 5 modalità: da 0 a 2 "altamente centrata sul candidato"; da 2,1 a 4 "prevalentemente centrata sul candidato"; da 4,1 a 6 "tra il partito e il candidato"; da 6,1 a 8 "prevalentemente centrata sul partito"; da 8,1 a 10 "altamente centrata sul partito".

2. Did you raise any local issues during your campaign?. Il candidato poteva rispondere sì o no a questa domanda. 
organismi politici a livello ministeriale, di giunta regionale o di consiglio regionale e 1 ' $11,30 \%$ dei candidati ha lavorato come dirigente/funzionario del proprio partito. La Norvegia, con il suo $42 \%$ di candidati precedentemente impiegati in staff di partito, e il 20\% di ex dirigenti o funzionari di partito, ci dimostra che in questo Paese, rispetto agli altri, questo passaggio risulta importante per l'avviamento alla carriera politica. Avere avuto incarichi politici istituzionali precedenti, come esser stato Deputato, Senatore, Sindaco o Presidente di provincia $(55,30 \%$ della selezione) o aver avuto incarichi di partito a livello comunale, provinciale, regionale o nazionale $(76,80 \%)$, è un passaggio piuttosto comune a tutti i candidati dei Paesi della nostra selezione. Nel Regno Unito solo il $21 \%$ degli intervistati dichiara di aver avuto precedenti incarichi politici, e si attesta sotto la media del campione anche per quel che attiene ai precedenti incarichi di partito. 


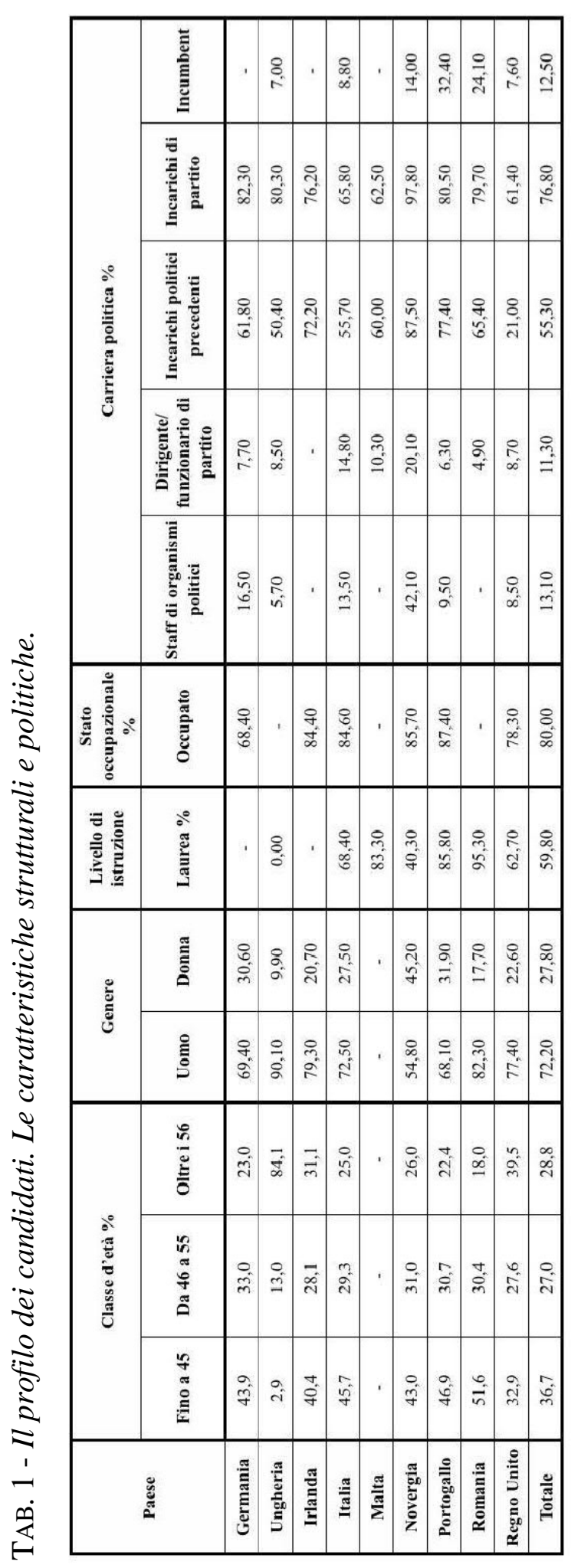


I dati relativi alla nostra analisi comparata saranno illustrati a partire dalla suddivisione tra Paesi che presentano candidate-based electoral systems, party-based electoral systems e mixed-members systems, al fine di analizzare la professionalizzazione e la personalizzazione delle campagne elettorali dei candidati alle elezioni generali.

Nello specifico verranno presentati i dati relativi all'indice di professionalizzazione delle campagne elettorali e quelli relativi alla personalizzazione della stessa, attraverso la presentazione dei temi e del profilo della campagna centrato sul candidato o sul partito.

I dati verranno letti confrontando i Paesi tra i tre gruppi, con la lente orientata alle regole del gioco che governano la competizione, ovvero i sistemi elettorali di ciascun Paese.

L'indice di professionalizzazione della campagna elettorale. - L'analisi dei diversi livelli di professionalizzazione delle campagne elettorali dei candidati, attraverso la presentazione dell'istogramma (cfr. Fig. 2), mostra un'evidente differenza tra i tre gruppi di Nazioni.

I livelli più alti di questo indice li ritroviamo nei quattro Paesi caratterizzati dalla presenza di candidate - based electoral systems. Al suo interno inoltre, notiamo livelli ancora maggiori in Irlanda e Malta, che, con il Single Trasferibile Vote, favoriscono una competizione non solo interna al partito, ma anche tra partiti concorrenti. In questi Paesi i candidati sembrano avere la necessità e l'esigenza di gestire la complessità della competizione elettorale con maggiore professionalità, facendo ricorso a servizi specialistici offerti da professionisti della comunicazione, sondaggisti ed esperti di marketing politico ed allungando i tempi della propria campagna elettorale. Anche in Ungheria il livello di professionalizzazione della campagna elettorale dei candidati raggiunge livelli alti. Questi sono leggermente più bassi in Germania, dove raggiunge un punteggio di 6,3. Interessante appare anche il risultato nei tre Paesi che presentano un sistema elettorale proporzionale con liste bloccate. Qui, vogliamo ricordarlo, la posizione in lista dei candidati è determinata dal partito, gli elettori esprimono un voto di preferenza per una lista di partito, non per un particolare candidato. In un sistema con liste bloccate, i partiti politici ricevono un numero di seggi proporzionale al numero di voti che ottengono attraverso una formula elettorale che applica il metodo del quoziente o divisore. Il primo seggio vinto dal partito va al candidato che occupa il primo posto in lista, il secondo seggio va al secondo candidato, e così via. I candidati limitano il ricorso ai consulenti politici e avvertono meno l'esigenza di dotarsi di uno staff professionalizzato. La campagna elettorale 
è per lo più condotta dal partito a livello nazionale. Spesso è il partito che, a livello centrale, affida la gestione e il coordinamento strategico della campagna elettorale ad agenzie e/o consulenti esterni legati al mondo del marketing politico. L'Italia, rispetto alla Norvegia e al Portogallo, presenta un indice di professionalizzazione leggermente più alto $(5,2)$. 


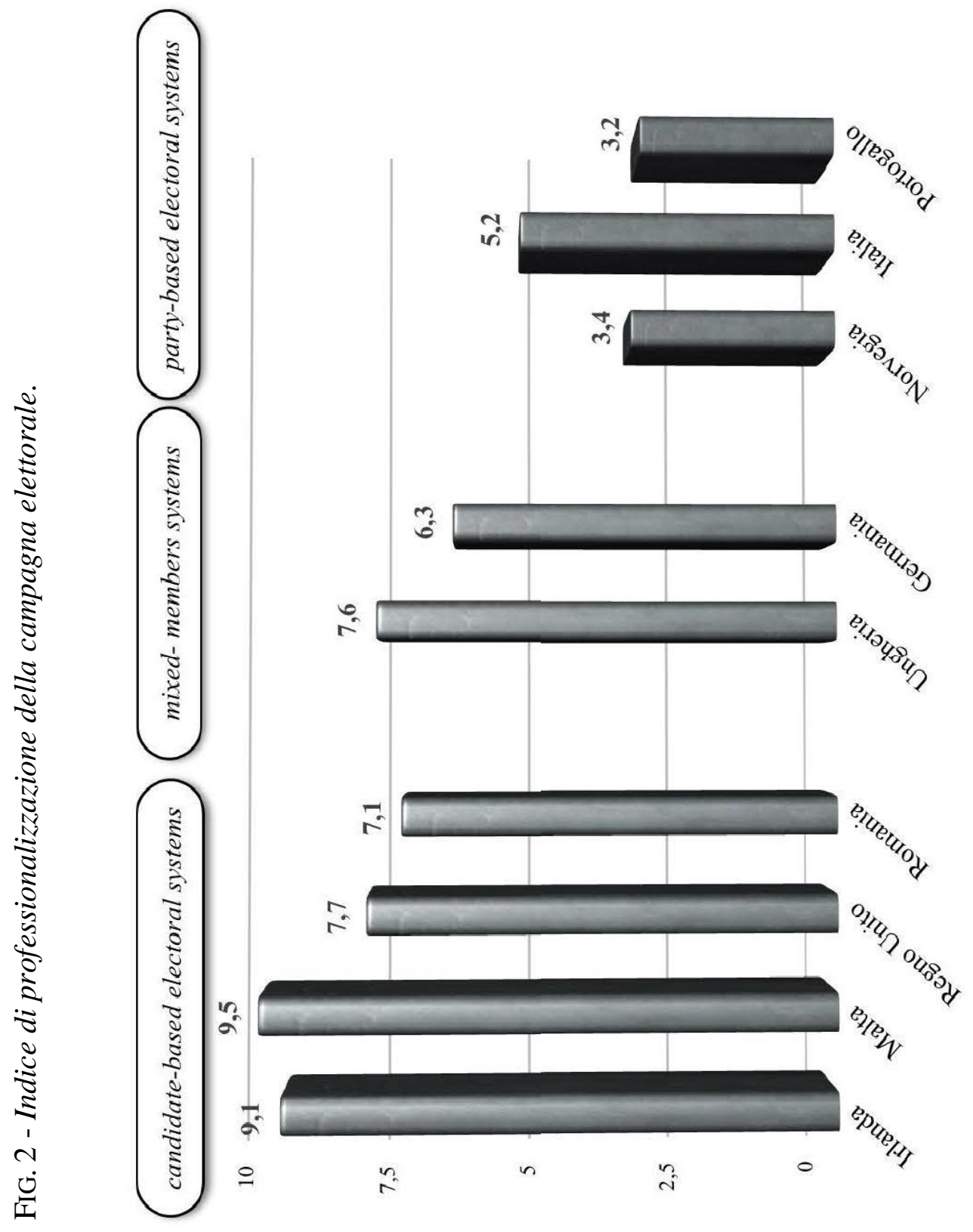


La personalizzazione della campagna elettorale. - Nelle campagne elettorali postmoderne si assiste sempre più ad una crescente personalizzazione della competizione, che sposta l'enfasi della comunicazione sul candidato sottraendo sempre più il controllo delle campagne ai partiti. Questo è vero in particolare in quei contesti in cui i sistemi elettorali sono centrati sul candidato, lo è meno in presenza di sistemi a liste bloccate in cui il partito politico esercita forti influenze. Questo assunto teorico lo riscontriamo nella distribuzione presentata in Figura 3 (cfr. Fig. 3).

I candidati ai Parlamenti dei Paesi della nostra selezione, dovendo descrivere l'obiettivo principale della propria campagna elettorale, indicando un punteggio su una scala che andava da 0 a 10 dove 0 significava "Attrarre maggiore attenzione possibile su di me come candidato" e 10 "Attrarre maggiore attenzione possibile sul partito", scelsero dei punteggi che ben si allineano al nostro presupposto di partenza.

Dei tre Paesi con party - based electoral system, in Norvegia e in Portogallo quasi il $90 \%$ dei candidati ha indicato che il suo principale obiettivo di campagna elettorale era quello di attrarre maggiore attenzione verso il proprio partito (esprimendo un valore superiore al 6). Intorno al $60 \%$ sia in Italia che in Germania. Per un candidato in una lista bloccata, la possibilità di essere eletto è determinata dal numero di seggi che il proprio partito riesce ad ottenere nella sua circoscrizione, dunque il suo obiettivo di campagna elettorale non può che essere quello di incrementare i voti del suo partito. I candidati di Paesi come l'Ungheria e l'Irlanda, hanno più degli altri caratterizzato il proprio profilo personale nel corso della campagna elettorale, spostando l'attenzione su loro stessi. Ad eccezione di Malta, anche per gli altri Paesi della selezione, che presentano sistemi elettorali centrati sul candidato, i livelli di attenzione maggiori li ritroviamo sul candidato e non sul partito. I casi mixed - member system sono di più difficile lettura. Questi sistemi, come abbiamo visto sopra, combinano circoscrizioni elettorali uninominali e liste bloccate. Le risposte andrebbero lette tenendo conto del tipo di competizione in cui erano inseriti i candidati. Purtroppo non disponiamo di dati che chiariscano se si trattava di collegi uninominali o plurinominali. 


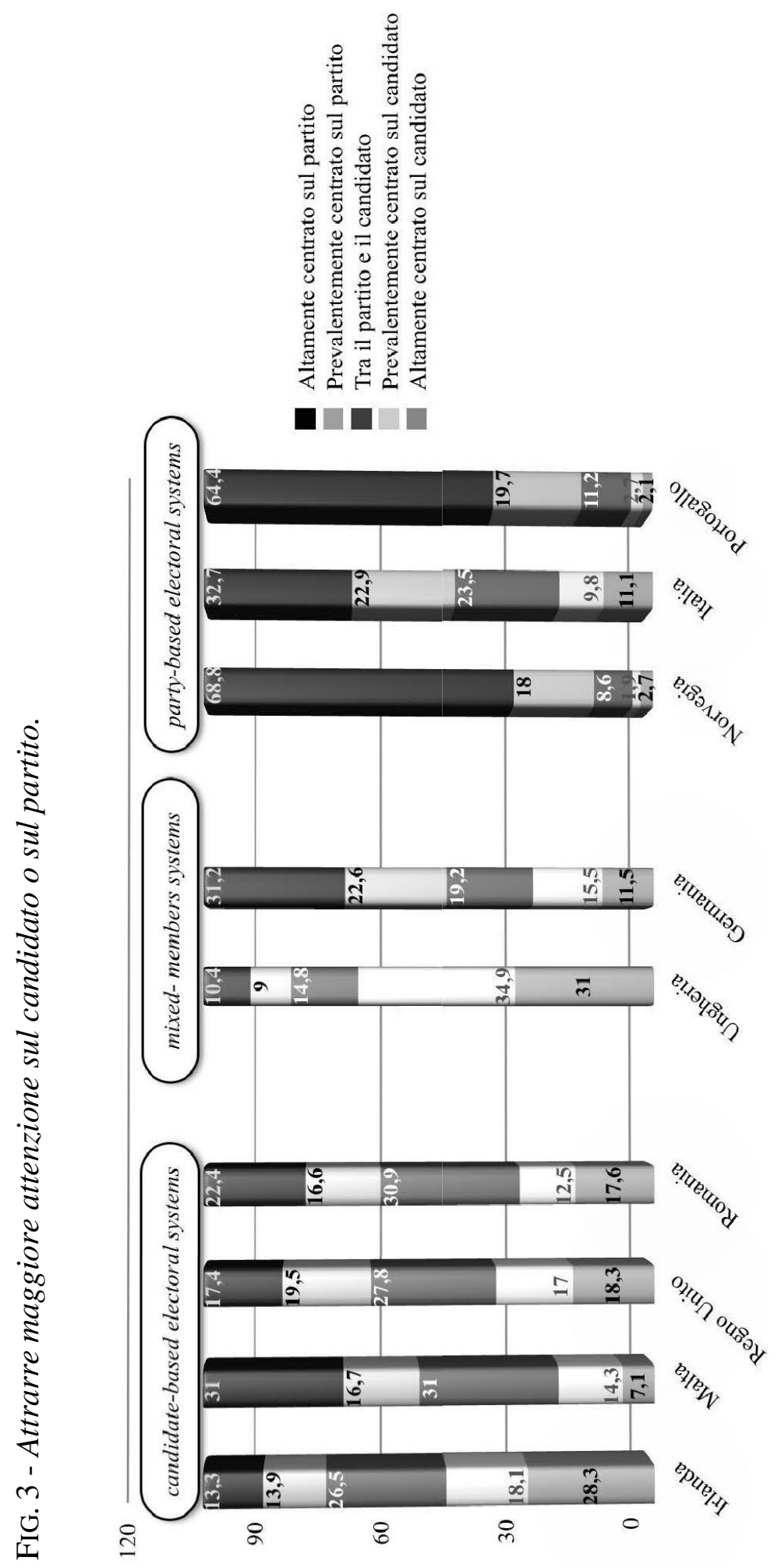


Un altro aspetto da considerare quando si valuta il livello di personalizzazione della campagna elettorale è certamente quello relativo ai temi (cfr. Fig. 4).

Il ricorso ai temi locali e a quelli del proprio collegio di appartenenza è maggiore tra $\mathrm{i}$ candidati nei Paesi in cui vi è un sistema elettorale basato sul candidato. L'unica eccezione è Malta, dove questo dato non è così netto. Molto probabilmente, date le limitate dimensioni territoriali e il carattere insulare, la differenziazione dei temi risulta limitata. In Norvegia e in Portogallo in particolare, con il suo $73,4 \%$, i candidati hanno la tendenza a sposare i contenuti della propria campagna elettorale sui temi di cui il partito si fa promotore a livello centrale. Questo dato è meno forte in Italia dove ben il 56\% dei candidati ha fatto dei temi locali e dei problemi del proprio territorio, l'argomento principale dei suoi contenuti in campagna elettorale. Nonostante non vi fosse la necessità di costruire un consenso personale, il candidato italiano non ha abbandonato del tutto il rapporto con il suo elettorato di riferimento, facendo una sintesi tra il programma di partito e i temi locali. La classe politica italiana probabilmente conserva quell'eredità che deriva dalla tradizione maggioritaria che ha caratterizzato per molti anni il suo sistema elettorale, attribuibile a quello che viene definito effetto "inerzia" dei sistemi elettorali (Denemark 2003, p. 615) o "resilienza" dello stile di campagna (Ma e Choy 2003, p. 363): i candidati - specie quelli già impegnati in precedenti tornate elettorali - tendono ad apporre resistenza nei confronti di un cambiamento di strategia che l'adozione di un nuovo sistema elettorale consiglierebbe.

Anche in questo caso la Germania e l'Ungheria sono di difficile lettura. Mentre la Germania sembra assimilabile al caso italiano, l'Ungheria è addirittura il Paese che più degli altri ha visto i suoi candidati concentrati sui problemi della propria circoscrizione elettorale.

In ultima analisi possiamo affermare che, anche il livello di personalizzazione della campagna elettorale, come quello di professionalizzazione, è legato ad elementi di tipo strutturale come il sistema elettorale di ciascun Paese. 


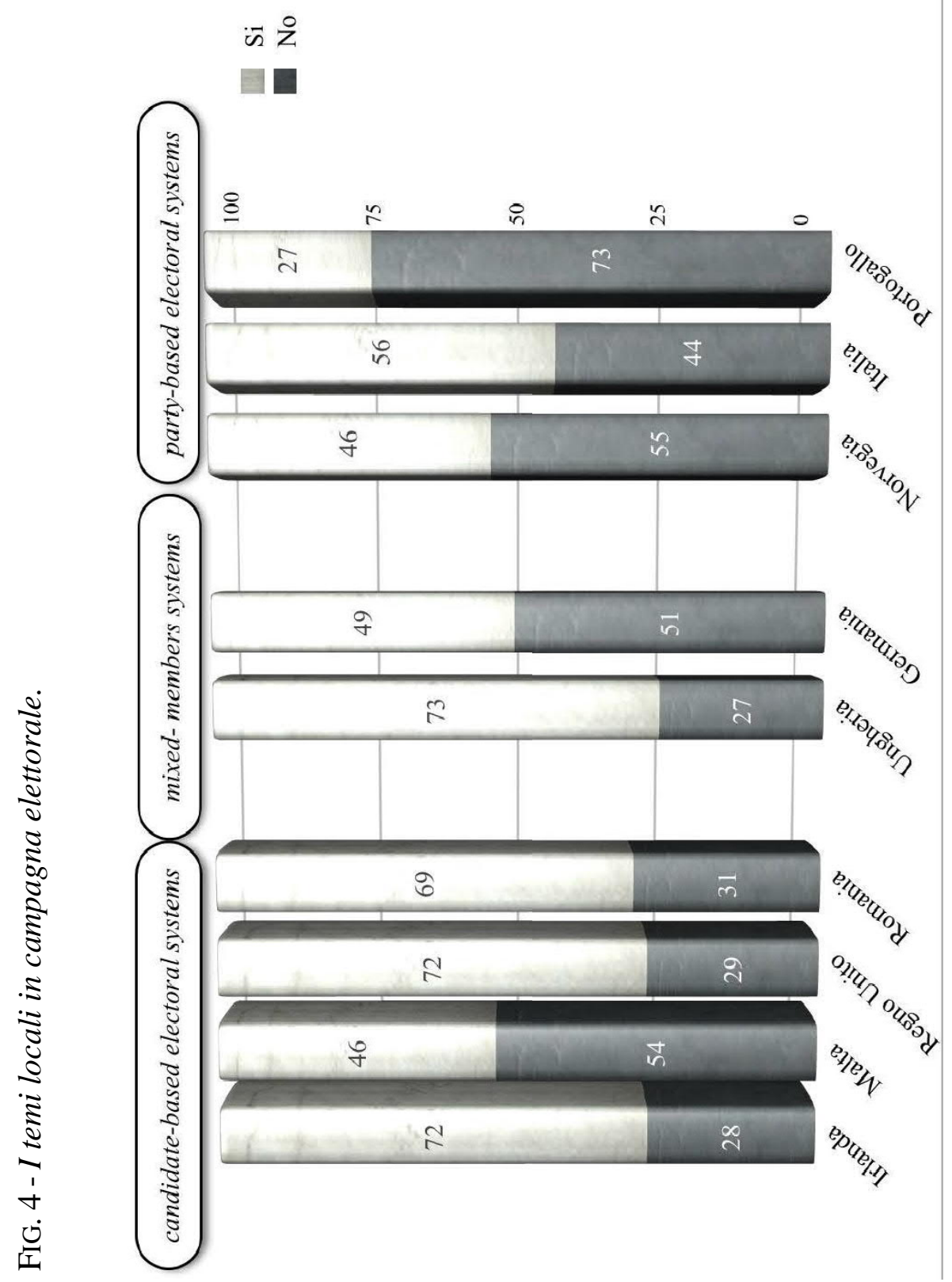

64 


\section{Modelli di campagna elettorale con l'analisi delle corrispondenze}

In questa sezione si cercherà di offrire un'efficace sintesi dei dati attraverso la rappresentazione simultanea di variabili con l'intento di analizzare le loro relazioni. Per compiere questa analisi multivariata utilizzeremo la tecnica dell'analisi delle corrispondenze multiple $(\mathrm{acm})$. L'obiettivo sarà quello di descrivere la nostra matrice dati riducendola a un numero minore di dimensioni, che esprimono delle combinazioni tra variabili. Queste dimensioni saranno in grado di riprodurre la maggior parte delle associazioni presenti fra le variabili analizzate in un ridotto numero di fattori che esprimono delle combinazioni tra le modalità originarie. Una volta individuati i fattori abbiamo rappresentato le variabili/modalità come punti proiettati all'interno di spazi fattoriali ortogonali fra loro.

Nell'analisi delle corrispondenze multiple le variabili/modalità si possono dividere in attive e supplementari: le prime concorrono direttamente alla formazione degli assi fattoriali, le seconde non contribuiscono alla formazione dei fattori ma sono utilizzate, attraverso la loro collocazione sugli assi fattoriali, per interpretare $\mathrm{i}$ fattori e per studiare eventuali legami di interdipendenza con questi.

Nell'analisi grafica dell'acm la nuvola di punti che rappresenta le variabili/modalità (sia attive sia supplementari) attraverso le loro coordinate fattoriali, illustreranno a partire dalla loro posizioni rispetto agli assi e le distanze tra i punti stessi, la struttura delle relazioni fra le variabili/modalità e fra queste e i fattori. Più un punto è lontano dall'origine di un asse, maggiore è il suo contributo alla formazione dell'asse stesso. Più elevata è la prossimità tra due variabili/modalità, maggiore è l'attrazione fra loro (Di Franco, 2001).

Fatta questa premessa presentiamo le variabili utilizzate per la nostra acm: Country, ovvero il Paese in cui si sono svolte le general election oggetto della nostra analisi, che come sappiamo si distinguono per il tipo di sistema elettorale; la variabile "Attenzione della campagna elettorale rivolta al partito o al candidato" (Att_Party_Candidate) e la variabile relativa alla presenza dei temi locali tra i contenuti della campagna elettorale (Local_issues), per gli aspetti relativi alla personalizzazione della campagna; ed infine, le variabili sui tempi di campagna che abbiamo illustrato nel dettaglio nel paragrafo metodologico (Camp_FullTime, Camp_Start, Camp_Hours) e quelle relative allo staff, ovvero l'ampiezza e la presenza di consulenti (Consultants e Staff_Size), per gli aspetti relativi alla professionalizzazione della campagna. Tutte le variabili/modalità 
nell'analisi sono state trattate come attive, eccezion fatta per la variabile Country che svolge nella nostra acm una funzione suppletiva.

Prima di illustrare i risultati della nostra analisi delle corrispondenze multiple, sembra opportuno presentare il coefficiente che ne sintetizza l'attendibilità. L'alpha di Cronbach descrive la coerenza interna di raggruppamenti di variabili. In generale elevati valori di alpha indicano che i soggetti esaminati esprimono un atteggiamento coerente riguardo a ciascun item appartenente a ciascuna dimensione. La verifica della coerenza interna di ogni subtest permette di approfondire lo studio e la definizione della struttura fattoriale. I ricercatori sono concordi nell'adottare il valore di alpha $=.60$ come riferimento di un livello accettabile di coerenza interna. Il livello di attendibilità della nostra analisi presenta nella prima dimensione il valore alpha di Cronbach 0.60, mentre nella seconda dimensione 0.41.

Entrando adesso nel vivo della nostra analisi, presentiamo il grafico di categoria congiunto (Fig. 5).

FIG. 5 - L'analisi delle corrispondenze multiple.

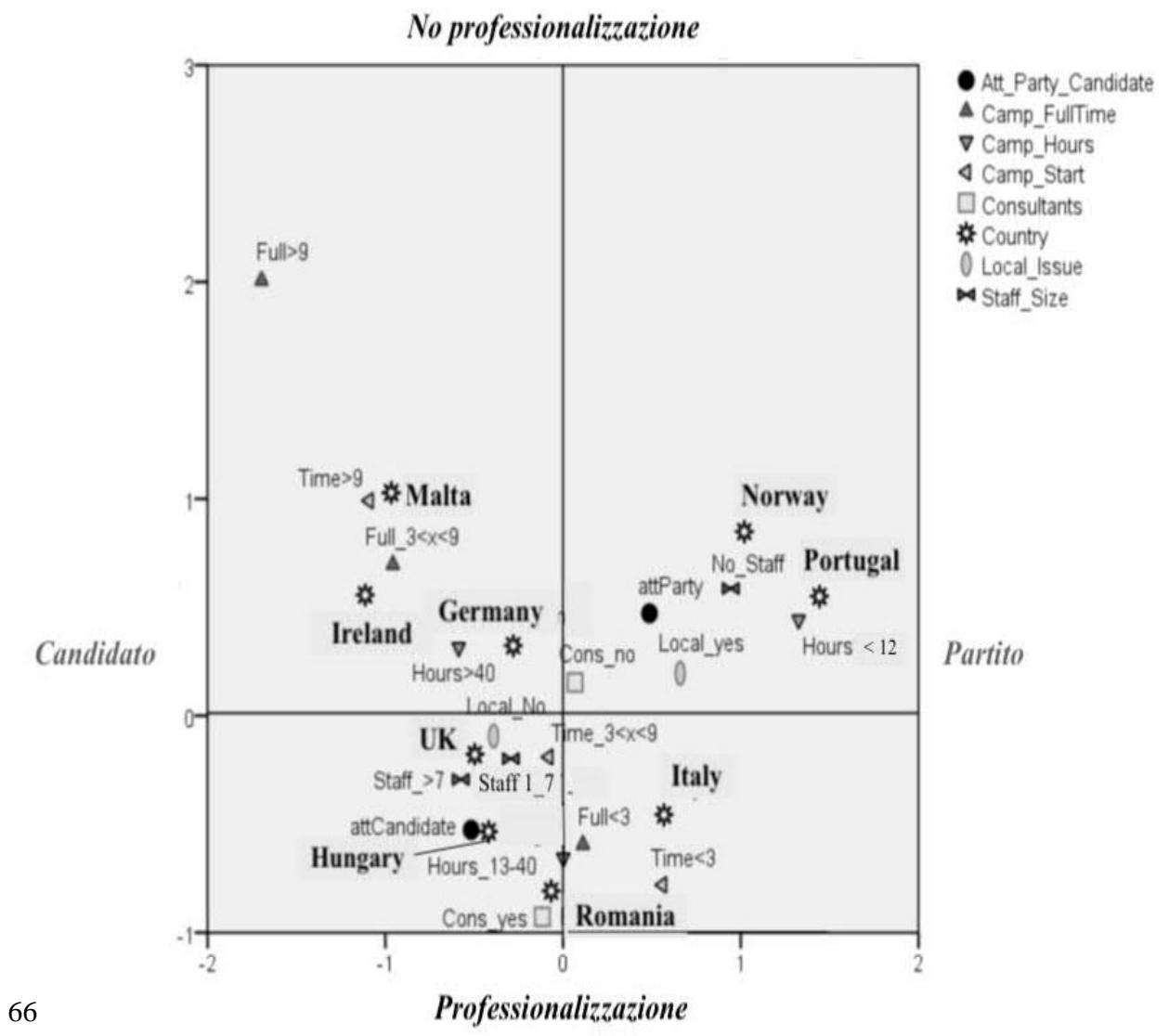


In questo grafico la distribuzione dei punti sul piano rappresenta il peso di ciascuna variabile rispetto ad ogni dimensione. Il diagramma di dispersione evidenzia le variabili che più contribuiscono alla formazione della dimensione.

La posizione di ogni variabile sul diagramma dipende dai contributi assoluti e relativi dalle coordinate fattoriali delle variabili/modalità attive che contribuiscono alla costruzione dei fattori e dalle coordinate fattoriali e i valori test delle variabili/modalità illustrative associate con il fattore. Di solito, le variabili/modalità attive caratterizzate da valori alti nelle coordinate fattoriali (quelle più distanti dall'origine degli assi) sono quelle che contribuiscono di più alla formazione dell'asse stesso. Al contrario, la posizione della variabile sarà prossima al baricentro ${ }^{7}$.

Fatte queste premesse analizziamo la soluzione che restituisce il nostro diagramma di dispersione: l'asse delle ascisse presenta la contrapposizione tra modalità di variabili legate ad una campagna candidato-centrica (-) e modalità legate ad una campagna partito-centrica (+) (Att_Party_Candidate). Sempre sul primo asse si trovano su fronti opposti campagne intense con un impegno diretto del candidato nelle attività (-) e attività di mobilitazione più limitate che richiedono un numero di ore settimanali inferiore alla dozzina (+) (Camp_Hours). In quest'ultimo caso - probabilmente per l'impegno diretto dell'organizzazione del partito gli staff personali sono assenti. Al contrario sulla polarità negativa del primo asse, troviamo la presenza di staff ampi e compositi (Staff_Size); sull'asse delle ordinate si concentrano delle modalità di variabile che entrano nel merito dell'approccio alle campagne elettorali, contrapponendo quelle lunghe e fin da subito intense (Camp_FullTime e Camp_Start), con un impegno diretto del candidato a quelle brevi e guidate da consulenti politici (Consultants_Yes).

\footnotetext{
${ }^{7}$ Se due o più variabili sono vicine fra loro e collocate presso un'estremità di un semiasse rappresentante una componente, ciò indica che esse sono altamente correlate in modo positivo fra loro e con la dimensione in questione (in modo positivo o negativo a seconda che si tratti del semiasse positivo o negativo della dimensione). Se due variabili si distanziano dal baricentro, ma una sul semiasse positivo e l'altra su quello negativo di una stessa dimensione, ciò indica che tra le due variabili esiste un'alta correlazione negativa e che entrambe presentano pesi elevati su quella dimensione (peso negativo per la variabile che si trova sul semiasse negativo e positivo per l'altra). Se due variabili, o due gruppi di variabili sono una vicina ad un'asse e l'altra al secondo asse (non importa in quale quadrante del piano) ciò indica che tra le due variabili, o gruppi di variabili, ci sono basse correlazioni e che esse presentano pesi alti su una dimensione e bassi sull'altra.
} 
Il plot evidenzia diversi possibili modelli di campagna elettorale ma soprattutto mette in luce come $\mathrm{i}$ vincoli normativi siano interpretati all'interno di culture politiche diverse. Nel primo quadrante troviamo le tracce di una campagna di lungo periodo, cominciata in molti casi anche un anno prima delle elezioni, e con un rilevante coinvolgimento del candidato nelle attività di mobilitazione: non solo la campagna fulltime inizia in largo anticipo rispetto alla data delle elezioni, ma anche il numero di ore spese nelle attività è ingente, superiore alle quaranta nell'ultima settimana. Queste variabili - fortemente associate con Paesi caratterizzati da un sistema elettorale candidato-centrico (Malta ed Irlanda) - denotano solo alcuni aspetti delle campagne postmoderne e in particolare la dilatazione dei tempi (Blumenthal, 1980). L'altro aspetto centrale appare collegato - come vedremo successivamente - al terzo quadrante nel quale si osserva il requisito della professionalizzazione degli staff. Più vicina all'origine degli assi è la Germania che è fortemente associata con la presenza di campagne labour-intensive per il candidato.

Nel secondo quadrante abbiamo un tipo di campagna poco intensa (nell'ultima settimana i candidati si dedicavano alle attività di propaganda per meno di 12 ore), più sensibile alle logiche di partito, in cui il candidato nelle attività di mobilitazione assume al più un ruolo di comprimario. Appare coerente la mancanza di staff personali e ancora di più, di consulenti politici. Questa associazione tra variabili è contestualizzabile nella logica dei sistemi elettorali con liste bloccate presente in Portogallo e Norvegia nei quali una gestione professionalizzata della campagna elettorale non risulta necessaria, per gli esiti spesso scontati della competizione: la vittoria del seggio è legata alla performance del partito, e più ancora, alla posizione in lista dei candidati.

Questo modello di campagna appare speculare a quello del terzo quadrante, fortemente caratterizzato dalla presenza dei consulenti e da organizzazioni ampie - in alcuni casi vere e proprie war rooms - che concentrano gli sforzi sulla valorizzazione del candidato. Queste variabili si associano a Paesi come la Romania e il Regno Unito, caratterizzati da sistemi elettorali con collegi uninominali, in cui la competizione interpartitica coinvolge direttamente i singoli candidati. L'immagine personale del candidato è importante tanto quanto il profilo del partito, consigliando quindi una valorizzazione delle caratteristiche individuali e una campagna elettorale personalizzata. Per motivi diversi Regno Unito e Romania hanno sviluppato un' "industria" della consulenza politica, anche se di genesi diversa: nel primo caso si tratta di agenzie e di singoli consulenti che si sono sviluppati a fianco dei partiti o dei singoli uomini 
politici, guidando il superamento di momenti di crisi in una prospettiva di lungo periodo e l'approccio alla digitalizzazione; nel secondo caso si tratta di un sistema che si dota di consulenza politica, attingendo a professionisti che vengono dall'estero, in particolare statunitensi, chiamati ad affiancare $i$ candidati in un contesto di debole istituzionalizzazione dei partiti (LessMarshment e Lilleker, 2012; Schafferer, 2006). Questo approdo alla consulenza riguarda anche l'Ungheria che ritroviamo nel terzo riquadro, anche se in presenza di un sistema elettorale misto.

Nel quarto quadrante - fortemente associato con l'Italia - troviamo alcune modalità che denotano tempi di durata della campagna piuttosto corti e un'intensità delle attività che si manifesta solo negli ultimi tre mesi. In questo contesto ritroviamo anche una parziale professionalizzazione delle campagne che appare poco in linea con la presenza di liste bloccate e che potrebbe essere riconducibile ad una sedimentazione dell'esperienza maggioritaria come già ricordato.

\section{Conclusioni}

I sistemi elettorali sembrano in grado di condizionare le campagne elettorali dei candidati, producendo modelli differenti al variare dei tipi di voto. La nostra osservazione delle campagne elettorali privilegia il punto di vista del candidato che è il protagonista nel caso di candidate-based electoral systems e un semplice comprimario nei party-based electoral systems. In quest'ultimo caso il peso del candidato sulla scelta dell'elettore è ininfluente, specie in presenza di liste bloccate lunghe. Il ruolo del candidato nel primo caso spiega invece la necessità di una forte caratterizzazione dell'immagine personale, ottenuta con il supporto di uno staff di advisors e con una strategia di personalizzazione del messaggio.

Nella nostra selezione sono presenti Paesi con sistemi elettorali molto diversi fra di loro. Dall'analisi di questa selezione di casi, con la consapevolezza delle difficoltà derivanti da un approccio comparativo (Esser e Strömbäck, 2012), emerge la centralità del sistema elettorale per comprendere la natura delle attività di mobilitazione ma che, tuttavia, questo elemento non è in grado di spiegarne l'intera variabilità.

Procedendo per gradi, abbiamo osservato delle linee di tendenza riguardo professionalizzazione e personalizzazione.

L'analisi dell'indice di professionalizzazione evidenzia un quadro piuttosto differenziato tra i Paesi Europei inseriti nella nostra analisi. Come atteso, i livelli più alti di questo indice si riscontrano nei quattro Paesi caratterizzati dalla presenza di candidate - based electoral systems, dove $\mathrm{i}$ 
livelli di competizione tra i partiti e tra i candidati dello stesso partito (per i sistemi con il voto singolo trasferibile) sono maggiori. Staff politici ampi, caratterizzati dalla presenza di consulenti politici e la dilatazione dei tempi, sembrano contraddistinguere le campagne elettorali in questi Paesi. Diversamente, in quei Paesi che adottano un sistema elettorale caratterizzato dalla presenza di liste bloccate, ritroviamo una professionalizzazione leggera, che prevede raramente la presenza di grandi staff che gestiscono "chiavi in mano" le campagne elettorali.

Anche l'analisi della personalizzazione mostra delle regolarità: i Paesi con sistemi elettorali centrati sul candidato tendono a personalizzare la propria campagna elettorale e recuperano i temi locali; diversamente, tra i candidati dei Paesi con sistemi elettorali basati sul partito la tendenza è quella di non accentuare il proprio profilo personale in campagna elettorale e di attribuire più importanza alla comunicazione riguardante il partito, inoltre questi limitano il ricorso ai temi locali e spostano i contenuti della campagna sulle issues sostenute dal partito a livello centrale.

Tra questi Paesi l'Italia sembra fare la differenza, almeno per quanto attiene gli aspetti relativi alla personalizzazione della campagna elettorale. Evidentemente l'aver avuto per molti anni un sistema elettorale differente, con un impianto prevalentemente di tipo maggioritario, ha portato la classe politica italiana a mantenere un rapporto diretto con l'elettorato del proprio territorio ${ }^{8}$.

I casi mixed-member systems sono di più difficile lettura. Questi sistemi, combinando prevalentemente circoscrizioni elettorali uninominali e

\footnotetext{
${ }^{8}$ L'effetto "inerzia" non spiega tuttavia la variabilità in termini di professionalizzazione e personalizzazione tra le campagne elettorali italiane che, in un precedente studio ad hoc su 150 parlamentari italiani nella XVII Legislatura, è stato possibile evidenziare. È necessario provare a riflettere criticamente sul concetto stesso di campagna elettorale uscendo dalla prospettiva dell'immediata ricerca del voto: le sue attività possono essere, per il candidato, un modo per accreditare una leadership su un territorio o all'interno di un partito, o essere il battistrada per successive mobilitazioni, considerando la campagna solo un viatico per obiettivi di lungo periodo. Questo potrebbe spiegare l'impegno (apparentemente poco razionale) dei candidati in campagne dall'esito scontato. Da questa precedente ricerca, emerge ad esempio che sono i candidati in "water mark" ad intensificare la campagna personale per l'incertezza dell'elezione che produce un investimento maggiore in termini di risorse impiegate. Inoltre incide anche la concezione individuale di rappresentanza nel parlamento: chi pensa di rappresentare gli interessi del territorio e di costruire un futuro politico a partire da questo, utilizza la campagna elettorale per rafforzare la propria posizione, mentre chi gioca la sua partita su un "collegio nazionale", puntando sulla qualità del lavoro parlamentare e sui ruoli assunti nella leadership di partito, considera la mobilitazione individuale come un elemento accessorio, di rilevanza limitata.
} 
liste bloccate, presentano dei risultati a cavallo tra i Paesi che si collocano ai due estremi dell'ideale continuum che abbiamo descritto in premessa.

L'analisi multivariata delle dimensioni riconducibili alla postmodern campaign ci restituisce un quadro più complesso, indicando che queste dimensioni non sono necessariamente associate. Se è vero che i sistemi elettorali influenzano la campagna elettorale, chiaramente si mantengono spazi di variabilità determinati da elementi come il sistema partitico e la sua polarizzazione (Karp, 2012), l'ampiezza del collegio elettorale, le caratteristiche individuali del candidato e, come ricordano Gibson e Römmele, le caratteristiche interne al partito (Gibson e Römmele 2001, p. 37). Nell'acm le variabili che erano state utilizzate per la costruzione dell'indice di professionalizzazione e quelle collegate alla personalizzazione, sono state valutate congiuntamente. A questo proposito è interessante notare che le variabili che nella riflessione sulle campagne elettorali postmoderne sono tradizionalmente legate risultano scarsamente associate. Queste ultime dovrebbero evidenziare un allungamento dei tempi e un'accentuata personalizzazione e professionalizzazione. Dai risultati del nostro studio emerge che l'allungamento e l'intensificazione delle attività (specie del candidato) si legano a campagne poco professionalizzate mentre viceversa quelle professionalizzate hanno una durata meno elevata e, come atteso, hanno degli staff di campagna allargati. Nelle prime il tempo impiegato sembrerebbe compensare la mancanza di una specifica professionalità nella gestione. Quello che accomuna i sistemi candidatecentred è, in ogni caso, una intensità elevata delle attività che si concretizza secondo modalità diverse che, è ipotizzabile, dipendano anche dalla cultura politica e dai partiti presenti. 


\section{Riferimenti bibliografici}

BenNetT W. L. e SEgERberg A. [2012], «The logic of connective action. Digital media and the personalization of contentious politics», in Information, Communication \& Society, 15, 5, pp. 739-768.

BLUMENTHAL S. [1980], The permanent campaign, New York, Simon \& Schuster.

BORDIGNON F. [2014], Il partito del capo. Da Berlusconi a Renzi, Sant'Angelo di Romagna, Maggioli.

BORMANN N.C. e GOLDER M. [2013], «Democratic electoral systems around the world, 1946-2011», in Electoral Studies, 32(2), pp. 360-369.

CALISE M. [2010], Il partito personale. I due corpi del leader, RomaBari, Laterza.

CAmatarri S. e Segatti P. [2016], «Stili di rappresentanza politica», in Segatti P. e Di Virgilio A. (a cura di), La rappresentanza politica in Italia. Candidati ed elettori nelle elezioni politiche del 2013, Bologna, Il Mulino, pp. 265-294.

CAREY J. M. e SHUGART M. [1995], «Incentives to cultivate a personal vote: A rank ordering of electoral formulas», in Electoral Studies, 14, 4, pp. 417-439.

CARTER E. e FARRELl D.M. [2010], «Electoral Systems and Election Management», In LeDuc L., Niemi R.G., Norris P, (eds), Comparing Democracies, Sage Publications Ltd.

Clark W., Golder R.M., e Golder S.N. [2011], Principi di scienza politica, Milano, McGraw-Hill.

COLOMER J. M. [2017], « Party System Effects on Electoral Rules», in Herron E., Pekkanen R. e Shugart M. S. (eds), The Oxford Handbook of Electoral Systems, Political Science, Parties and Bureaucracy, Political Institutions, Online Publication.

Cox G.W. [1999], «Electoral rules and calculus of mobilization» in Legislative Studies Quarterly, 24, 3, pp. pp. 387- 420.

Cox G. W. [2015], « Electoral Rules, Mobilization, and Turnout», in Annual Review of Political Science, 18,1, pp. 49-68.

DENEMARK D. [2003], «Electoral Change, Inertia and Campaigns in New Zealand: The First Modern FPP Campaign in 1987 and the First MMP Campaign in 1996», in Party Politics, 9, 5, pp. 601-18.

DI FRANCO G. [2001], EDS: Esplorare descrivere e sintetizzare $i$ dati, Milano, FrancoAngeli. 
ESSER F. e STRÖMBÄCK J. [2012], «Comparing Election Campaign Communication», in Esser F. e Hanitzsch T. (eds), The Handbook of Comparative Communication Research, New York, Routledge.

FARREL D. M. e WEBB M. [2002], «Political parties as campaign organizations», in Russell J. D. e Wattenberg M. P. (eds), Parties without partisans: political change in advanced industrial democracies, Oxford Scholarship Online.

GIBSON R. e RÖMMELE A. [2001], «A party centered theory of professionalized campaigning», in Harward International Journal of Press Politics, 6, 4, pp. 31- 43.

Karp J. A., Banducci S. A. e Bowler S. [2007], «Getting Out the Vote: Party Mobilization», in Comparative Perspective - British Journal of Political Science, 38, 1, pp. 91-112.

KARP J. A. [2012], «Electoral Systems, Party Mobilisation and Political Engagement», in Australian Journal of Political Science, 47, 1, pp. 71-89.

KREISS D. [2015], «Digital Campaigning», in Freelon D. e Coleman S. (eds), Handbook of Digital Politics, New York, Edgar Elgar.

Lago I., Bermúdez S., GuinJoAn M., Rowe K. e SimON P. [2019], «Party Mobilization and Electoral Systems», in Government and Opposition, vol. 54, 1, pp. 1-24.

LEES-MARSHMENT J. e LILLEKER D. [2012], «Knowledge sharing and lesson learning: consultants' perspectives on the international sharing of political marketing strategy», in Contemporary Politics, 18, 3, pp. 343-354.

Lijphart A. [1994], Electoral System and Party Sistem. A Study of Twenty-Seven Democracies 1945-1990, Oxford, Oxford University Press.

MA N. e CHOY C. [2003], «The Impact of Electoral Rule Change on Party Campaign Strategy: Hong Kong as a Case Study», in Party Politics, 9, 3, pp. 347- 367.

MANCINI P. [2015], «New Frontiers in Political Professionalism», in Political Communication, 16, 3, pp. 231 - 245.

Müller W. C. e Strøm K. [1999], Policy, Office or Votes? How Political Parties in Western Europe Make Hard Decisions, Cambridge, Cambridge University Press.

Negrine R., Holtz-Bacha C., Papathanassopoulos S. e Mancini P. [2007], The Professionalisation of Political Communication, Bristol, Intellect.

Panebianco A. [1982], Modelli di Partito. Organizzazione e potere nei partiti politici, Bologna, Il Mulino. 
PINTO L. e VERZICHELLI L. [2016], «La "scelta prima della scelta": profilo dei candidati, selezione e percorsi di carriera politica», in Segatti P. e Di Virgilio A. (a cura di), La rappresentanza politica in Italia. Candidati ed elettori nelle elezioni politiche del 2013, Bologna, Il Mulino, pp. 61- 89.

Plasser F. e Plasser G. [2002], Global political Campaigning. A Worldwide Analysis of Campaign Professionals and Their practices, Praeger, Westport, Connecticut.

POGUNTKE T. e WeBB P. [2005], The presidentialization of politics in Democratic Societies, Oxford, Oxford University Press.

Russo F. [2013], Gli Onorevoli. Cosa fanno e come ci rappresentano i nostri parlamentari, Bologna, Il Mulino.

Sampugnaro R. [2006], Dentro la campagna. Partiti, candidati e consulenza politica, Acireale, Bonanno.

SAMPUGNARO R. [2010], "Vicine eppur diverse. L'influenza delle regole sulle campagne e sulla mobilitazione: due competizioni a confronto», in Fusaro C. (a cura di), Governi locali e regionali in Europa fra sistemi elettorali e scelte di voto, Firenze, SISE, pp. 699-724.

SCHAFFERER C. [2006], «The Globalisation of Political Marketing: An Introduction», in Schafferer C. (eds), Election Campaigning in East and Southeast Asia: Globalisation of Political Marketing, Aldershot, Ashgate.

SHUGART M. [2001], «Electoral "efficiency" and the move to mixed member systems», in Electoral Studies, 20, pp. 173-193.

SHUGART M. e WATTENBERG M. [2001], «Mixed-Member electoral systems: a definition and a tipology», in Mixed-Member electoral systems: the best of both worlds?, Oxford, Oxford University Press.

STRÖMBÄCK J. e KIOUSIS S. [2014], «Strategic Political Communication in Election Campaigns», in Political Communication, Mouton de Gruyter, Carsten Reinemann, pp.109-128.

TENSCHER J., MYKKÄNEN J. e MORING T. [2012], «Modes of Professional Campaigning A Four-Country Comparison in the European Parliamentary Elections», in The International Journal of Press/Politics, 17, 2, pp. 145-168.

TENSCHER J. e MYKKÄNEN J. [2014], «Two Levels of Campaigning: An Empirical Test of the Party-Centred Theory of Professionalisation», in Political Studies, 1, pp. 20-41.

Tenscher J., KoC-Michalska K., Lilleker D. G., MYKKÄNEN J., WAlter A. S., FindOR A., JALALi C. e RÓKA J. [2015], «The professionals speak: Practitioners' perspectives on professional election campaigning», in European Journal of Communication, vol. 31, 2, pp. 95-119. 